\title{
Effectiveness of Sodium Azide Alone Compared to Sodium Azide in Combination with Methyl Nitrosurea for Rice Mutagenesis
}

\author{
Thomas H. Tai ${ }^{1,2} *^{\dagger}$, Areum Chun ${ }^{3 \dagger}$, Isabelle M. Henry ${ }^{4}$, Kathie J. Ngo ${ }^{4}$, Diana Burkart-Waco ${ }^{1,2}$ \\ ${ }^{1}$ USDA-ARS Crops Pathology and Genetics Research Unit, Davis, CA 95616, USA \\ ${ }^{2}$ Department of Plant Sciences, University of California, Davis, CA 95616, USA \\ ${ }^{3}$ Crop Post-harvest Technology Research Division, National Institute of Crop Science, Rural Development Administration, Suwon \\ 16429, Korea \\ ${ }^{4}$ Genome Center and Department of Plant Biology, University of California, Davis, CA 95616, USA
}

\begin{abstract}
Rice seeds of the temperate japonica cultivar Kitaake were mutagenized with sodium azide alone and in combination with methyl nitrosourea. Using the reduced representation sequencing method Restriction Enzyme Sequence Comparative Analysis (RESCAN), the mutation densities, types and local sequence context were determined in the resulting M2 generation mutants. The results indicate that sodium azide is as effective alone as in combination with methyl nitrosourea in generating mutations in rice. In both cases, GC>AT transitions were the predominant mutation type and similar local sequence contexts were observed (5'-G-G-R-3' for sodium azide, 5'-R-G-R-3' for sodium azide plus methyl nitrosourea). Differences in local sequence context with those reported for another common chemical mutagen, ethyl methanesulfonate, suggests that rice mutant populations developed by combining mutants derived independently from sodium azide and ethyl methanesulfonate may exhibit a broader spectrum of mutations and mutant phenotypes.
\end{abstract}

Keywords Chemical mutagenesis, Reduced representation sequencing, Mutation density, Sodium azide, Methyl nitrosourea, Rice

\section{INTRODUCTION}

Modern plant mutation breeding can be traced back to the pioneering work of L.J. Stadler on the mutagenic effects of x-rays on maize, barley, and wheat (Stadler 1928; Stadler 1930). From 1930 to 1970, researchers in many countries began employing both physical and chemical agents to induce useful traits in an array of crop species.
Notable among the earliest efforts were those of A. Gustafsson of Sweden, whose work on barley mutagenesis provided much of the impetus for the improvement of plant mutation techniques (Kharkwal 2012). In the late 1950s, the first rice varieties developed directly from induced mutation were released in China and a few years later the Chinese produced the first rice variety derived by breeding with an induced rice mutant (Rutger 1992). Today, the

\footnotetext{
Received September 27, 2016; Revised November 10, 2016; Accepted November 12, 2016; Published November 30, 2016

*Comesponding author Thomas H. Tai, Thomas.Tai@ars.usda.gov, Tel: +1-530-752-4342, Fax: +1-530-754-7195

${ }^{\dagger}$ These authors contributed equally to this work as co-first authors.

${ }^{\dagger}$ Present address: Department of Plant Sciences, University of California, Davis, CA 95616, USA.

Mention of trade names or commercial products in this publication is solely for the purpose of providing specific information and does not imply recommendation or endorsement by the U.S. Department of Agriculture. USDA is an equal opportunity provider and employer.
} 
FAO/IAEA Mutant Variety Database (https://mvd.iaea. org/) lists over 800 rice varieties that have been develop directly by mutagenesis or from breeding with mutant germplasm. Induced mutagenesis has remained an important tool for plant breeders as it is considered to be a traditional method and the resulting varieties are not viewed with the concerns associated with transgenic or recombinant DNA-based techniques.

Over the past 15 years or so, induced mutagenesis has regained the attention of basic researchers entering a post-genome sequencing era where dissecting gene function is center stage. Among the functional genomics approaches being employed, the reverse genetics method Targeting of Induced Local Lesions in Genomes (TILLING) was specifically developed to exploit the genetic variation induced by chemical mutagenesis (McCallum et al. 2000). TILLING is particularly suited for species recalcitrant to genetic transformation, and thus not amenable to insertional mutagenesis (Comai and Henikoff 2006). Another benefit of chemical mutagenesis for functional studies is the possibility of producing mutant alleles with altered function rather than complete knockouts (Comai and Henikoff 2006). Generation of an "allelic series" could provide important information on gene function. Rice functional genomics has greatly profited from mutant populations derived by insertional mutagenesis (Wei et al. 2013), but a number of useful induced mutagenesis resources have also been developed (Kurata et al. 2005; Tai 2007; Wei et al. 2013). These induced mutant resources have been primarily generated using chemical mutagens such as ethyl methanesulfonate (EMS), diepoxybutane, sodium azide, and methyl nitrosourea (MNU) (Kurata et al. 2005; Wu et al. 2005; Till et al. 2007; Suzuki et al. 2008; Wei et al. 2013).

For reverse genetics methods like TILLING, mutant populations with optimal mutation densities are needed in order to facilitate efficient, cost-effective screening (Till et al. 2007; Monson-Miller et al. 2012). In rice, TILLING was initially performed using mutant populations selected based on the frequency of mutant phenotypes (e.g., embryo lethality, chlorophyll mutations) although these may not accurately reflect the number or density of lesions at the DNA level. Initial attempts to generate TILLING populations in rice with acceptable levels of mutations were of limited success (Wu et al. 2005). Although subsequent work led to the establishment of a rice TIILING population with a suitable mutation density (Till et al. 2007), the usefulness of evaluating the mutant populations at the DNA level prior to investing in development of large populations became evident. With the availability of relatively low-cost, ultra-high-throughput sequencing, various approaches for rapid and cost-effective assessment of mutation density (Monson-Miller et al. 2012) and mutation discovery (Tsai et al. 2011; Henry et al. 2014) have been developed.

In this study, we have employed a reduced representation sequencing method called Restriction Enzyme Sequence Comparative Analysis (RESCAN) (Monson-Miller et al. 2012) to compare the mutation densities and types generated in rice using two mutagenesis protocols involving the chemical mutagen sodium azide $\left(\mathrm{NaN}_{3}\right)$. Previously, a double seed mutagenesis protocol consisting of sodium azide treatment followed by MNU treatment was used to develop part of a rice mutant population (Till et al. 2007) based on the efficacy of this combination of mutagens in barley (Szarejko and Maluszynski 1999). Our objective was to determine if mutagenesis with sodium azide alone produced comparable results to those of the double mutagenesis using $\mathrm{NaN}_{3}$ followed by MNU.

\section{MATERIALS AND METHODS}

\section{Plant materials and chemical mutagenesis}

Oryza sativa ssp. japonica (cv. Kitaake) seeds were subjected to chemical mutagenesis using three treatments: sodium azide $\left(\mathrm{NaN}_{3}\right)$ only, $\mathrm{NaN}_{3}$ plus $\mathrm{MNU}$, and $\mathrm{MNU}$ only. Mutagenesis was carried out on batches of about 500 seeds (based on weight) with three replications for each mutagenic treatment and one untreated control. All steps involving seed soaking and washing were performed in standard $250 \mathrm{ml}$ hybridization bottles using a hybridization oven (Robbins Scientific, Sunnyvale, CA, USA).

Seeds were soaked in $100 \mathrm{ml}$ of ultrapure water for 16 hours at $\sim 25^{\circ} \mathrm{C}$ (tube rotator speed setting 10) prior to treatment. After draining, $20 \mathrm{ml}$ of $0.1 \mathrm{M}$ sodium phosphate 
buffer ( $\mathrm{pH} 3$ ) was added to each batch of seeds ( $19.8 \mathrm{ml}$ for those to be treated with $\mathrm{NaN}_{3}$ ). For treatments involving $\mathrm{NaN}_{3}, 0.2 \mathrm{ml}$ of $100 \mathrm{mM} \mathrm{NaN}_{3}$ (prepared just prior to use) was added to each seed batch for a final concentration of 1 $\mathrm{mM}$. Seeds (including no treatment control and MNU only) were incubated as before for 3 hours. All batches were subjected to three 5 minutes washes with $50 \mathrm{ml}$ of ultrapure water. After the final wash, $50 \mathrm{ml}$ of ultrapure water was added and seeds were incubated overnight ( $\sim 18$ hours). After draining, $19 \mathrm{ml}$ of ultrapure water was added to the seed batches to be treated with MNU and $1 \mathrm{ml}$ of freshly prepared $300 \mathrm{mM}$ MNU was added for a final concentration of $15 \mathrm{mM}$. Twenty $\mathrm{ml}$ of ultrapure water was added to each of the remaining batches (control and $\mathrm{NaN}_{3}$ only treatment). All seeds were incubated for 3 hours followed by washing as described above. After the final wash, $50 \mathrm{ml}$ of ultrapure water was added and each seed batch was incubated in the oven for 4 hours and then drained.

Each batch of drained seeds was transferred to separate $150 \mathrm{~mm}$ plastic petri dishes lined with paper towels and 10 $\mathrm{ml}$ of ultrapure water was added before sealing and placing in a growth chamber at $\sim 25^{\circ} \mathrm{C}$. After two days, the seeds were counted and transferred to $10 \times 20 \mathrm{~cm}$ trays containing UC Davis greenhouse soil mix $\mathrm{C}$ and grown in the greenhouse. Seedlings were counted after 7 days and transplanted to 50 cell plug flats to facilitate production of M2 seeds, which were harvested 85 to 90 days later.

\section{DNA extraction, construction and sequencing of RESCAN libraries}

Total genomic DNA was isolated from wild type Kitaake and M2 individuals using a potassium acetatesodium dodecyl sulfate method (Tai and Tanksley 1990) with minor modifications. Fresh leaf tissue from 4-6 week old plants was flash frozen and ground to a fine powder in the presence of liquid nitrogen prior to the extraction. Reduced representation sequencing was performed using the RESCAN method (Monson-Miller et al. 2012; Kim and Tai 2013).

RESCAN libraries were constructed from each individual as described previously (Kim and Tai 2013) with modifications (Burkart-Waco et al. 2016). For each library, $500 \mathrm{ng}$ of genomic DNA was digested with the restriction enzyme NlaIII (CATG $\downarrow$; NEB, Ipswich, MA, USA) for $>6$ hours at $37^{\circ} \mathrm{C}$. After verifying complete digestion by agarose gel electrophoresis, the digestion products were purified using an equal volume of AMPure XP reagent (Beckman Coulter Genomics, Danvers, MA, USA). After washing and eluting DNA from AMPure XP beads in $51 \mu$ of elution buffer ( $10 \mathrm{mM}$ Tris- $\mathrm{HCl}, \mathrm{pH} 8.0)$, $50 \mu \mathrm{l}$ of the digested and cleaned DNA was transferred to a new $0.2 \mathrm{ml}$ tube.

Libraries were constructed using a KAPA LTP Library Preparation Kit for Illumina ${ }^{\circledR}$ platforms (KAPA Biosystems, Inc., Wilmington, MA, USA) and Nextflex ${ }^{\text {TM }}$ DNA 8-bp barcodes (Bioo Scientific, Austin, TX, USA) according to the manufacturer's protocol. Ligations were carried out in $50 \mu \mathrm{l}$ reactions and $50 \mu \mathrm{l}$ of polyethylene glycol (PEG)/ $\mathrm{NaCl}$ solid-phase reversible immobilization (SPRI) solution was added to each reaction followed by binding, washing, and elution in $100 \mu \mathrm{l}$ of elution buffer. Size selection was performed by adding $60 \mu \mathrm{l}$ of PEG/NaCl SPRI solution to the ligation products in elution buffer with beads from the prior step. After pipetting to thoroughly mix, samples were incubated at room temperature for 5 to 15 minutes to allow DNA fragments larger than $450 \mathrm{bp}$ to bind to the SPRI beads. After clearing the samples on a magnetic stand, $155 \mu \mathrm{l}$ of the supernatant was transferred to a clean tube. Twenty microlite of AMPure XP beads were added to the supernatants and mixed well by pipetting and samples were incubated for 5 to 15 minutes to allow fragments $>250 \mathrm{bp}$ to bind to the beads. Samples were cleared on a magnetic stand, supernatants were removed by pipetting, and the beads were washed according to manufacturer's instructions. Size selected DNAs (250-450 bp) were eluted from the beads in $25 \mu$ of elution buffer.

Polymerase chain reaction enrichment was performed in $50 \mu \mathrm{l}$ reactions consisting of $20 \mu \mathrm{l}$ of size selected DNAs, $25 \mu \mathrm{l}$ of 2X KAPA mix (KAPA LTP Library Preparation Kit; (KAPA Biosystems, Inc.), and $5 \mu$ of primers (10 $\mu \mathrm{M})$. The amplification program used was: $98^{\circ} \mathrm{C}$ for 45 seconds, 10 to 12 cycles of $98^{\circ} \mathrm{C}$ for 15 seconds, $60^{\circ} \mathrm{C}$ for 30 seconds, and $72^{\circ} \mathrm{C}$ for 30 seconds, followed by $72^{\circ} \mathrm{C}$ for 1 minute. Enrichment (and size selection) were verified by running $4 \mu \mathrm{l}$ of the reactions on a $2 \%$ agarose/1X 
tris-acetate-ethylenediaminetetraacetic acid gel. RESCAN libraries were sequenced using two lanes (10 libraries per lane) of an Illumina HiSeq 2500 (SR50) and sequencing was performed by the Vincent J. Coates Genomics Sequencing Laboratory at the University of California at Berkeley.

\section{Sequence processing and mutation discovery}

Illumina sequence reads were sorted according to their barcode sequence, which was trimmed from the reads. Next, reads were trimmed for quality using a custom python script called 'Allprep' (available for download at http://comailab. genomecenter.ucdavis.edu/index.php/Barcoded_data_prep aration_tools). The following parameters were used: the mean PHRED score over a 5 bp-window $\geq 20$ and processed sequence length $\geq 35 \mathrm{bp}$. The processed reads were then aligned to $O$. sativa Nipponbare genome build MSU 6.1 (http://rice.plantbiology.msu.edu/) using Burrows-Wheeler Aligner version 0.7.5a (Li and Durbin 2009), and using default mismatch parameters. Sam/bam files were generated using Samtools (Li et al. 2009) using an additional custom python script (http://comailab.genomecenter.ucdavis.edu/ index.php/ Bwa-doall). We used default parameters except that chimeric reads, i.e., reads that contained an NlaIII restriction site, were cut in silico and only the first part of the read was retained. The presence of a restriction site in a RESCAN sequencing read indicates either incomplete digestion by the restriction enzyme or ligation of two independent fragments, producing a chimeric read. Chimeric reads cause mis-mapping and erroneous SNPs. Next, data from all bam files were combined into a single mpileup file, using Samtools (Li et al. 2009) and default parameters (mapping quality $\geq 20$ ) and parsed using a third custom python script called mpileup-parser.py (http:// comailab.genomecenter.ucdavis.edu/index.php/Mpileup).

Mutation detection was performed using the Mutations and Polymorphisms Surveyor (MAPS) bioinformatics pipeline available at http://comailab.genomecenter. ucdavis.edu/index.php/MAPS. The specific parameters used for MAPS1 were as follows: -m m, -1 10, -c 20, -C 10000 , -I 10. The specific parameters for MAPS2 were: -c 20, -d 2, -p 20, -s 2, -1 10, - m m. Next, the percentage of C > T and $\mathrm{G}>\mathrm{A}$ mutations was calculated for different thresholds of mutation coverage, in order to estimate the rate of false positive mutations. A minimum coverage of 5 for the mutant allele was selected as it resulted in a percentage of canonical mutations $>90 \%$. Mutation rates were calculated based on coverage statistics specific to each library, as previously described (Henry et al. 2014).

To examine the local sequence context, the nucleotide frequencies in $20 \mathrm{bp}$ regions on each side of mutated $\mathrm{G}$ nucleotides (i.e., GC base pairs) were determined by retrieving $40 \mathrm{bp}$ of sequence surrounding the mutation site from the reference genome sequence. Another $40 \mathrm{bp}$ centered on the same nucleotide ( $\mathrm{G}$ or $\mathrm{C})$ were randomly chosen from 40-50 bp on either side of the mutation site to account for the possibility of local bias in sequence composition due to adjacent nucleotides. At each position, the relative percentage of the four nucleotides was determined, and the differences in percentages between the mutation sites and the random flanking sites were calculated.

\section{RESULTS}

\section{Seed mutagenesis of Kitaake}

Seed mutagenesis was performed on the temperate japonica variety Kitaake following a double mutagenesis protocol used previously to generate mutants in the temperate japonica variety Nipponbare for establishing a rice TILLING resource (Till et al. 2007). Seeds were treated initially with $1 \mathrm{mM} \mathrm{NaN}_{3}$ followed by $15 \mathrm{mM}$ MNU. For this study, mutagenesis was also performed by treating seeds with $1 \mathrm{mM} \mathrm{NaN}_{3}$ only and with $15 \mathrm{mM} \mathrm{MNU}$ only. All mutagenic treatments were conducted at the same time in triplicate and treatment with the single mutagens was performed to match the timing of the double mutagenesis protocol. Thus, in the case of the $\mathrm{NaN}_{3}$ only treatment, seeds were subjected to washing and incubation in water during the time that the MNU treatment was performed in the double mutagenesis protocol. Likewise, for the MNU only treatment, the initial incubation period was performed in water without $1 \mathrm{mM} \mathrm{NaN}_{3}$. The effects of the mutagenic treatments on seed germination and fertility of the first generation mutants (M1) are shown in Table 1. 
Table 1. Rice seed mutagenesis of the temperate japonica variety Kitaake.

\begin{tabular}{|c|c|c|c|c|c|c|}
\hline $\begin{array}{l}\text { Treatment } \\
\text { Batch No. }\end{array}$ & Mutagen & $\begin{array}{c}\text { No. seeds } \\
\text { mutagenized }\end{array}$ & $\begin{array}{l}\text { No. seeds } \\
\text { germinated }\end{array}$ & $\begin{array}{c}\text { Germination } \\
\text { rate }(\%)\end{array}$ & $\begin{array}{l}\text { No. fertile } \\
\text { M1 plant }\end{array}$ & $\begin{array}{c}\% \text { Survival } \\
\text { to } \text { seed }^{\mathrm{z})}\end{array}$ \\
\hline $\mathrm{K}-5$ & $\mathrm{NaN}_{3}$ & 499 & 395 & 79.2 & 354 & 89.6 \\
\hline K-6 & $\mathrm{NaN}_{3}$ & 501 & 480 & 95.8 & 393 & 81.9 \\
\hline $\mathrm{K}-7$ & $\mathrm{NaN}_{3}$ & 510 & 391 & 76.7 & 375 & 95.9 \\
\hline $\mathrm{K}-8$ & $\mathrm{NaN}_{3}+\mathrm{MNU}^{\mathrm{w})}$ & 498 & 365 & 73.3 & 362 & 99.2 \\
\hline $\mathrm{K}-9$ & $\mathrm{NaN}_{3}+\mathrm{MNU}$ & 506 & 365 & 72.1 & 365 & 100.0 \\
\hline $\mathrm{K}-10$ & $\mathrm{NaN}_{3}+\mathrm{MNU}$ & 509 & 411 & 80.7 & 369 & 89.8 \\
\hline $\mathrm{K}-2$ & MNU & 507 & 436 & 86.0 & 399 & 91.5 \\
\hline $\mathrm{K}-3$ & MNU & 501 & 436 & 87.0 & 403 & 92.4 \\
\hline $\mathrm{K}-4$ & MNU & 495 & 429 & 86.7 & 392 & 91.4 \\
\hline $\mathrm{K}-1$ & None & 513 & 441 & 86.0 & $185^{\mathrm{y})}$ & $100.0^{\mathrm{x})}$ \\
\hline
\end{tabular}

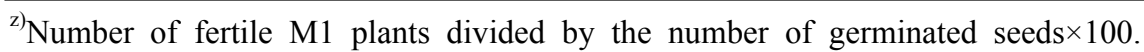

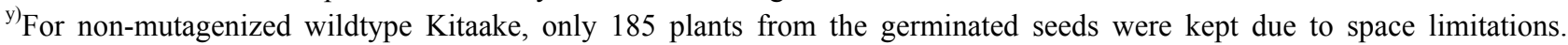

${ }^{\mathrm{x})}$ All of the 185 wildtype Kitaake plants produced seeds.

${ }^{\text {w) }} \mathrm{MNU}$ : methyl nitrosourea.

Table 2. Number of $\mathrm{Mb}$ assayed for mutations and number of mutations and mutation rates, asdetermined by RESCAN analysis.

\begin{tabular}{|c|c|c|c|c|c|c|c|c|c|c|}
\hline Library & Treatment & $\begin{array}{l}\text { Batch } \\
\text { No. }\end{array}$ & $\begin{array}{l}\text { Hom } \\
(\mathrm{Mb})^{\mathrm{z})}\end{array}$ & $\begin{array}{l}\text { Het } \\
(\mathrm{Mb})\end{array}$ & $\begin{array}{l}\text { Hom } \\
\text { Mut }^{\mathrm{y})}\end{array}$ & $\begin{array}{l}\text { Het } \\
\text { Mut }\end{array}$ & $\begin{array}{l}\text { Total } \\
\text { Mut }\end{array}$ & $\begin{array}{c}\text { Hom } \\
\text { Mut/Hom }^{\mathrm{x})}\end{array}$ & $\begin{array}{c}\text { Het } \\
\text { Mut/Het }\end{array}$ & $\begin{array}{l}\text { Mut rate } \\
\left.(\mathrm{Mut} / \mathrm{Mb})^{\mathrm{w}}\right)\end{array}$ \\
\hline KT16 & $\mathrm{NaN}_{3}$ & K-5 & 19.08 & 4.60 & 12 & 6 & 18 & 0.63 & 1.30 & 1.93 \\
\hline KT17 & $\mathrm{NaN}_{3}$ & $\mathrm{~K}-5$ & 45.32 & 35.15 & 58 & 57 & 115 & 1.28 & 1.62 & 2.90 \\
\hline KT21 & $\mathrm{NaN}_{3}$ & $\mathrm{~K}-6$ & 26.96 & 8.17 & 22 & 5 & 27 & 0.82 & 0.61 & 1.43 \\
\hline KT22 & $\mathrm{NaN}_{3}$ & $\mathrm{~K}-6$ & 33.06 & 16.08 & 33 & 14 & 47 & 1.00 & 0.87 & 1.87 \\
\hline KT26 & $\mathrm{NaN}_{3}$ & $\mathrm{~K}-7$ & 29.72 & 15.77 & 29 & 19 & 48 & 0.98 & 1.20 & 2.18 \\
\hline KT31 & $\mathrm{NaN}_{3}+\mathrm{MNU}$ & $\mathrm{K}-8$ & 30.63 & 13.68 & 20 & 21 & 41 & 0.65 & 1.53 & 2.19 \\
\hline KT32 & $\mathrm{NaN}_{3}+\mathrm{MNU}$ & $\mathrm{K}-8$ & 27.78 & 11.23 & 12 & 2 & 14 & 0.43 & 0.18 & 0.61 \\
\hline KT36 & $\mathrm{NaN}_{3}+\mathrm{MNU}$ & K-9 & 31.46 & 11.01 & 16 & 25 & 41 & 0.51 & 2.27 & 2.78 \\
\hline KT37 & $\mathrm{NaN}_{3}+\mathrm{MNU}$ & K-9 & 31.80 & 16.54 & 16 & 19 & 35 & 0.50 & 1.15 & 1.65 \\
\hline KT41 & $\mathrm{NaN}_{3}+\mathrm{MNU}$ & $\mathrm{K}-10$ & 36.22 & 22.50 & 11 & 27 & 38 & 0.30 & 1.20 & 1.50 \\
\hline KT1 & MNU & $\mathrm{K}-2$ & 54.72 & 28.30 & 1 & 9 & 10 & 0.02 & 0.32 & 0.34 \\
\hline KT2 & MNU & $\mathrm{K}-2$ & 36.86 & 18.66 & 0 & 0 & 0 & 0.00 & 0.00 & 0.00 \\
\hline KT6 & MNU & $\mathrm{K}-3$ & 51.58 & 36.14 & 0 & 1 & 1 & 0.00 & 0.03 & 0.03 \\
\hline KT7 & MNU & K-3 & 10.21 & $1.98^{\mathrm{v})}$ & 0 & 0 & 0 & 0.00 & N/A & 0.00 \\
\hline KT11 & MNU & $\mathrm{K}-4$ & 57.84 & 40.10 & 3 & 9 & 12 & 0.05 & 0.22 & 0.28 \\
\hline KT51 & None & $\mathrm{K}-1$ & 43.88 & 30.80 & 0 & 0 & 0 & 0.00 & 0.00 & 0.00 \\
\hline KT52 & None & $\mathrm{K}-1$ & 48.24 & 36.12 & 0 & 2 & 2 & 0.00 & 0.06 & 0.06 \\
\hline KT53 & None & $\mathrm{K}-1$ & 51.99 & 38.85 & 1 & 4 & 5 & 0.02 & 0.10 & 0.12 \\
\hline KT54 & None & $\mathrm{K}-1$ & 52.38 & 29.46 & 1 & 10 & 11 & 0.02 & 0.34 & 0.35 \\
\hline KT55 & None & K-1 & 38.67 & 11.66 & 0 & 1 & 1 & 0.00 & 0.09 & 0.09 \\
\hline
\end{tabular}

${ }^{\mathrm{z})}$ Number of sequenced bases assayed to identify homozygous mutations, Mb: mega base pairs.

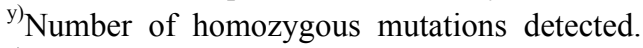

${ }^{\mathrm{x})}$ Mutation rate for homozygous mutations equals number of homozygous mutations divided by the number of sequenced bases assayed to identify those mutations.

${ }^{\text {w) }}$ Total Mutation rate is sum of homozygous and heterozygous mutation rates.

${ }^{v)}$ Insufficient number of sequenced bases to confirm the presence of heterozygous mutations.

${ }^{u}$ RESCAN: Restriction Enzyme Sequence Comparative Analysis, MNU: methyl nitrosourea, Hom: homozygous, Het: heterozygous, Mut: mutation, N/A: not applicable. 
The average germination rate (\%) of the mutagenized seeds over the three replications was $83.9 \% \pm 10.4 \%, 75.4 \% \pm 4.7 \%$, and $86.6 \% \pm 0.5 \%$ for the $\mathrm{NaN}_{3}$ only, $\mathrm{NaN}_{3}$ plus $\mathrm{MNU}$, and MNU only treatments, respectively. Of those germinating seeds, the average percentage surviving to produce M2 seeds over the three replications was $89.1 \% \pm 7.0 \%$, $96.3 \% \pm 5.7 \%$, and $91.8 \% \pm 0.6 \%$ for the $\mathrm{NaN}_{3}$ only, $\mathrm{NaN}_{3}$ plus MNU, and MNU only treatments. From seed to seed (M0 to M2), the average percentages of survival were $74.3 \% \pm 3.8 \%, 72.4 \% \pm 0.3 \%$, and $79.4 \% \pm 0.9 \%$, for $\mathrm{NaN}_{3}$ only, $\mathrm{NaN}_{3}$ plus $\mathrm{MNU}$, and $\mathrm{MNU}$ only, respectively.

\section{Evaluation of mutation type and density using RESCAN}

To evaluate the effectiveness of each of the mutagenic treatments, RESCAN libraries were constructed from five randomly selected mutants per treatment type (i.e., $\mathrm{NaN}_{3}$ only, $\mathrm{NaN}_{3}$ plus $\mathrm{MNU}$, and MNU only). In addition, libraries were prepared from five of the Kitaake wildtypes, which were subjected to the double mutagenesis protocol using water only. RESCAN libraries were sequenced on an Illumina HiSeq 2500 sequencer using an SR50 read type. The average number of sequence reads per library ranged from 12.1 million for the MNU only treatment libraries to 22.1 million for the wild type Kitaake and on average 96\% to $97 \%$ of the reads aligned to the reference genome ( $O$. sativa Nipponbare MSU 6.1) (Supplementary Table S1).
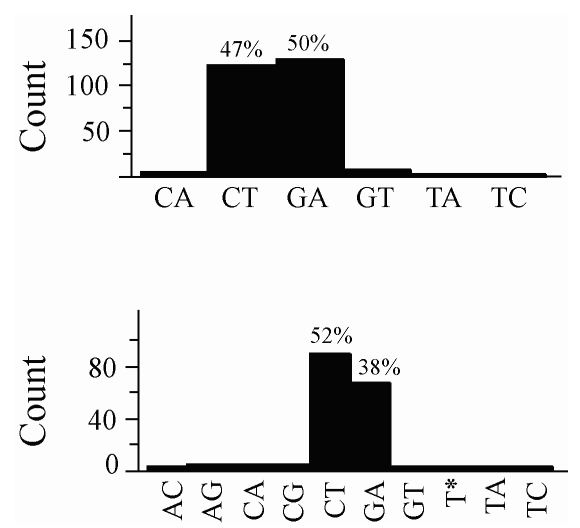

Fig. 1. Mutation types detected in $\mathrm{NaN}_{3}$ only (top) and $\mathrm{NaN}_{3}$ plus methyl nitrosourea (bottom) treatments. $\mathrm{CT}$ and GA mutation represent mutations on a guanine residue located either on the top strand $(\mathrm{G}$ to A mutation) or the bottom strand ( $\mathrm{C}$ to $\mathrm{T}$ mutation) of the reference sequence.
Analysis of the sequence reads revealed that the M2 mutants from the $\mathrm{NaN}_{3}$ only treatment had a mutation rate ranging from 1.4 to 2.9 mutations per $\mathrm{Mb}$ whereas the $\mathrm{NaN}_{3}$ plus MNU mutagenesis generated mutation rates from 0.6 to 2.8 mutations per $\mathrm{Mb}$ (Table 2). The number of mutations detected in the MNU only ( 0 to 0.34 mutations per $\mathrm{Mb}$ ) were essentially the same as the Kitaake wild type samples ( 0 to 0.35 mutations per $\mathrm{Mb}$ ) indicating that treatment of the germinated rice seeds with MNU did not generate a significant number of mutations.

Examination of the types of changes present among the mutations identified were consistent with previous reports that $\mathrm{NaN}_{3}$ mutagenesis yields predominantly $\mathrm{GC}>\mathrm{AT}$ transitions (Talamè et al. 2008; Monson-Miller et al. 2012). The mutations in the $\mathrm{NaN}_{3}$ only and $\mathrm{NaN}_{3}$ plus $\mathrm{MNU}$

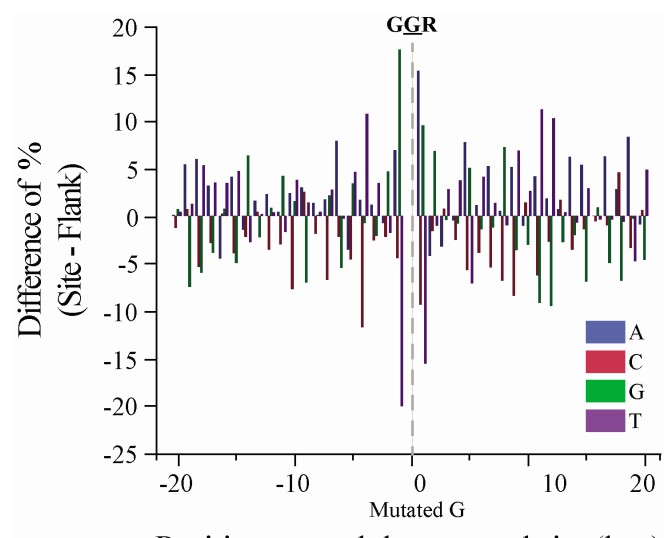

Position around the mutated site (bps)

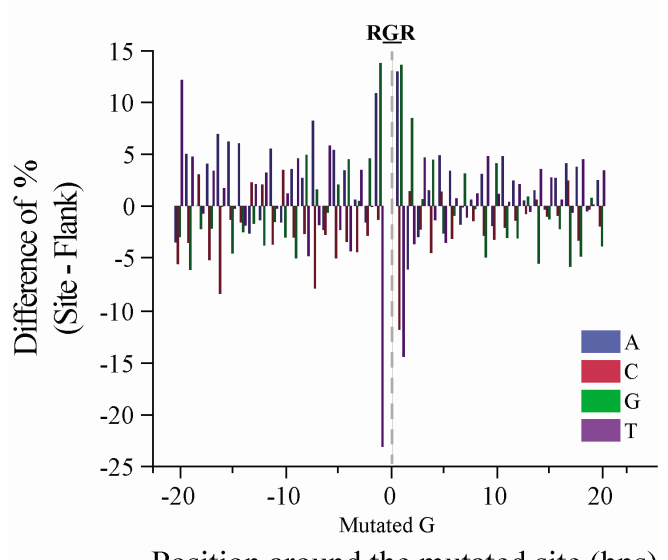

Position around the mutated site (bps)

Fig. 2. Analysis of nucleotide frequencies around the $\mathrm{NaN}_{3}$ only (top) and $\mathrm{NaN}_{3}$ plus methyl nitrosourea (bottom) treatments. $\mathrm{R}$ represent $\mathrm{G}$ or $\mathrm{A}$. 
mutants were overwhelming GC $>$ AT transitions (Fig. 1). The nucleotides immediately adjacent to the mutated bases (i.e., mutated $\mathrm{GC} \mathrm{bp)} \mathrm{were} \mathrm{examined} \mathrm{in} \mathrm{comparison} \mathrm{to}$ randomly selected GC bp within the local region (i.e., 40-50 bp flanking the mutation sites) to determine if the $\mathrm{NaN}_{3}$ and $\mathrm{NaN}_{3}$ plus MNU mutagens preferentially targeted specific sequence motifs (Fig. 2). Although the "noise" level was high due to the relatively low numbers of mutations, the patterns that were obtained clearly suggested a bias for a 5'-G-G-R-3' triplet (R represents $\mathrm{G}$ or A) in the case of $\mathrm{NaN}_{3}$ only. Similarly, the $\mathrm{NaN}_{3}$ plus MNU treatment showed a bias for a 5'-R-G-R-3' triplet.

\section{DISCUSSION}

Recent advances in ultra-high-throughput sequencing and computational approaches have provided a platform to leverage traditional tools such as induced mutagenesis for gene discovery and functional analysis (Tsai et al. 2011; Abe et al. 2012; Henry et al. 2014; Schneeberger 2014). Developing suitable mutant populations is a key component of this strategy. As such, identification of mutagens and mutagenic treatment protocols that optimize mutation densities in the species of interest enables smaller populations to be developed, reducing the costs associated with both screening (i.e., forward and reverse genetics) and maintaining (i.e., storage and rejuvenation of seeds) those populations.

Sodium azide was first identified as a powerful mutagen in barley in the early 1970s (Nilan et al. 1973). Soon thereafter, rice researchers found sodium azide to be an effective agent for seed mutagenesis (Harn and Lee 1975; Sarma et al. 1979; Awan et al. 1980; Hasegawa and Inoue 1980). In more recent times, sodium azide has been used to generate barley mutants (Talamè et al. 2008) and in combination with MNU to generate rice mutants (Till et al. 2007) for TILLING. Rice mutants derived from seed mutagenesis with sodium azide were used to develop and test the utility of RESCAN, a reduced representation sequencing-based strategy, in assessing mutation density using both reference genome-dependent and genomeindependent mutation discovery (Monson-Miller et al.
2012).

In this study, a modified version of RESCAN (Burkart-Waco et al. 2017) was employed to compare the mutation densities generated in rice mutants using sodium azide and sodium azide in combination with MNU. The double mutagenesis ( $\mathrm{NaN}_{3}$ plus MNU) protocol previously employed to generate rice mutants for TILLING (Till et al. 2007) was used with water substituting for the $\mathrm{MNU}\left(\mathrm{NaN}_{3}\right.$ only) and the sodium azide (MNU only) for comparison. Our results indicated that the $\mathrm{NaN}_{3}$ only and $\mathrm{NaN}_{3}$ plus MNU treatments yielded a similar range of mutation densities with the upper values around 3 mutations per $\mathrm{Mb}$. Overall, these densities were consistent with those observed previously for sodium azide mutagenesis of Kitaake (Monson-Miller et al. 2012) but lower than those reported for the $\mathrm{NaN}_{3}$ plus MNU mutagenesis of Nipponbare, which was estimated to be about 3.8 mutations per $\mathrm{Mb}$ but may be as low as 2 per $\mathrm{Mb}$ (Till et al. 2007). These differences might be due to differences in the varieties used although both are temperate japonicas. Also, the mutation density for the Nipponbare mutants was based on TILLING of 10 target genes rather than a genome-wide assessment. Mutagenesis with MNU only yielded essentially the same mutation densities observed in the water only (i.e., untreated) Kitaake wild types. While MNU is known to be a potent mutagen in rice (Suzuki et al. 2008), this result was not surprising as the MNU was added at the same time as the MNU in the double mutagenesis treatment. At that point, all the rice seeds had initiated germination (T. Tai, unpublished observation) so that the number of genetically effective cells (i.e., cells developing into germ line cells) would have increased significantly over those present originally in the dormant seeds. Thus, any MNU-induced mutations in a given cell would be less likely to be passed on to the M2 generation that was assayed by sequencing (i.e., these mutations would be somatic mutations). On the other hand, the sodium azide treatments reduced the growth rate of the emerging coleoptiles and radicles which were shorter when the MNU treatment was applied in the $\mathrm{NaN}_{3}$ plus MNU samples, than the MNU only and water only treatments (T. Tai, unpublished observation).

Evaluation of the types of mutations obtained from the 
various treatments indicated that both $\mathrm{NaN}_{3}$ only and $\mathrm{NaN}_{3}$ plus MNU resulted in almost exclusively $\mathrm{GC}>\mathrm{AT}$ transitions ( $90 \%$ or more of the mutations detected). This result is consistent with the effects of $\mathrm{NaN}_{3}$ on barley and rice (Talamè et al. 2008; Monson-Miller et al. 2012) and of MNU on rice (Suzuki et al. 2008). An examination of the nucleotides flanking the mutation sites (i.e., mutated GC bp) revealed very similar triplet sequences, 5'-G-G-R-3' for $\mathrm{NaN}_{3}$ only and 5'-R-G-R-3' for $\mathrm{NaN}_{3}$ plus MNU. A previous study on the local sequence context of MNUinduced rice mutants revealed the triplet 5'-R-G-N-3' (where $\mathrm{N}$ is any base) in $88 \%$ of the GC $>$ AT substitutions analyzed (Suzuki et al. 2010) perhaps confirming a stronger influence of $\mathrm{NaN}_{3}$ on the number of mutations generated in the $\mathrm{NaN}_{3}$ plus MNU treatment. Recently, an extensive characterization of the mutation sites in EMS-mutagenized rice revealed a strong bias for the 5'-R-G-C-3' triplet (Henry et al. 2014). This suggests that mutants generated with $\mathrm{NaN}_{3}$ might produce changes in genes differing from those derived from EMS mutagenesis and that populations developed from different mutagenic agents would be desirable.

Ultra-high-throughput sequencing-based detection of mutations facilitated the comparison of the mutation density, type, and local sequence context of mutation sites in rice mutants generated using chemical mutagenesis. Our results indicate that rice seed mutagenesis using $\mathrm{NaN}_{3}$ alone is as effective as a double mutagenesis in combination with MNU. Analysis of nucleotides flanking the mutation sites suggests that mutant populations consisting of $\mathrm{NaN}_{3}$ and EMS-derived mutants could provide a wider array of mutations and, therefore, mutant phenotypes. The approach employed here should be useful for evaluating the effectiveness of other mutagens and protocols for generating populations suitable for forward and reverse genetics.

\section{ACKNOWLEDGEMENTS}

This work was supported by the Rural Development Administration (RDA) of the Republic of Korea through the RDA/USDA-ARS Virtual Laboratory Program Project
No. PJ00923907 (A.C. and T.H.T.), USDA-ARS CRIS Project 5306-21000-021-00D (T.H.T.), and by the National Science Foundation Plant Genome Award DBI-0822383, TPGRP, "Efficient identification of induced mutations in crop species by ultra-high-throughput DNA sequencing" (to L. Comai). This work used the Vincent J. Coates Genomics Sequencing Laboratory at UC Berkeley, supported by NIH S10 Instrumentation Grants S10RR029668 and S10RR027303.

\section{REFERENCES}

Abe A, Kosugi S, Yoshida K, Natsume S, Takagi H, Kanzaki $\mathrm{H}$, et al. 2012. Genome sequencing reveals agronomically important loci in rice using MutMap. Nat. Biotechnol. 30: 174-178.

Awan MA, Konzak CF, Rutger JN, Nilan RA. 1980. Mutagenic effects of sodium azide in rice. Crop Sci. 20: 663-668.

Burkart-Waco D, Henry IM, Ngo K, Comai L, Tai TH. 2017. Determining mutation density using restriction enzyme sequence comparative analysis (RESCAN), p. 305-321. In: J. Jankowicz-Cieslak, T.H. Tai, J. Kumlehn, B.J. Till (ed.). Biotechnologies for plant mutation breeding. Springer International, Cham, Switzerland.

Comai L, Henikoff S. 2006. TILLING: Practical singlenucleotide mutation discovery. Plant J. 45: 684-694.

Harn C, Lee Y. 1975. Effect of sodium azide as mutagen in rice. Korean J. Breed. 30: 56-57.

Hasegawa H, Inoue M. 1980. Effects of sodium azide on seedling injury and chlorophyll mutation in rice. Jpn. J. Breed. 30: 301-308.

Henry IM, Nagalakshmi U, Lieberman MC, Ngo KJ, Krasileva KV, Vasquez-Gross H, et al. 2014. Efficient genome-wide detection and cataloging of EMS-induced mutations using exome capture and next-generation sequencing. Plant Cell. 26: 1382-1397.

Kharkwal MC. 2012. A brief history of plant mutagenesis, p. 21-30. In: Q.Y. Shu, B.P. Forster, H. Nakagawa (ed.). Plant mutation breeding and biotechnology. CAB International, Cambridge, MA.

Kim SI, Tai TH. 2013. Identification of SNPs in closely related temperate japonica rice cultivars using restriction enzyme-phased sequencing. PLoS ONE 8: e60176. 
Kurata N, Miyoshi K, Nonomura K, Yamazaki Y, Ito Y. 2005. Rice mutants and genes related to organ development, morphogenesis and physiological traits. Plant Cell. Physiol. 46: 48-62.

Li H, Durbin R. 2009. Fast and accurate short read alignment with Burrows-Wheeler transform. Bioinformatics 25: 1754-1760.

Li H, Handsaker B, Wysoker A, Fennell T, Ruan J, Homer N, et al. 2009. The sequence alignment/map format and SAMtools. Bioinformatics 25: 2078-2079.

McCallum CM, Comai L, Greene EA, Henikoff S. 2000. Targeted screening for induced mutations. Nat. Biotechnol. 18: 455-457.

Monson-Miller J, Sanchez-Mendez DC, Fass J, Henry IM, Tai TH, Comai L. 2012. Reference genome-independent assessment of mutation density using restriction enzymephased sequencing. BMC Genomics 13: 72.

Nilan RA, Sideris EG, Kleinhofs A, Sander C, Konzak CF. 1973. Azide-a potent mutagen. Mutat. Res. 17: 142-144.

Rutger JN. 1992. Impact of mutation breeding in rice. A review. Mut. Breed. Rev. 8: 3-23.

Sarma NP, Patnaik A, Jachuk PJ. 1979. Azide mutagenesis in rice - effect of concentration and soaking time on induced chlorophyll mutation frequency. Environ. Exp. Bot. 19: 117-121.

Schneeberger K. 2014. Using next-generation sequencing to isolate mutant genes from forward genetic screens. Nat. Rev. Genet. 15: 662-676.

Stadler LJ. 1928. Genetic effects of x-rays in maize. Proc. Natl. Acad. Sci. U.S.A. 14: 69-75.

Stadler LJ. 1930. Some genetic effects of x-rays in plants. J. Hered. 21: 3-19.

Suzuki T, Eiguchi M, Kumamaru T, Satoh H, Matsusaka H, Moriguchi K, et al. 2008. MNU-induced mutant pools and high performance TILLING enable finding of any gene mutation in rice. Mol. Genet. Genomics 279: 213-223.

Suzuki T, Moriguchi K, Tsuda K, Eiguchi M, Kumamaru T, Satoh H, et al. 2010. Neighboring nucleotide bias around MNU-induced mutations in rice. Rice Genet. Newsl. 25: 90-91.

Szarejko I, Maluszynski M. 1999. High frequency of mutations after mutagenic treatment of barley seeds with $\mathrm{NaN} 3$ and $\mathrm{MNH}$ with application of inter-incubation germination period. Mutat. Breed. Newsl. 44: 28-30.

Tai TH. 2007. Induced mutations in rice (Oryza sativa L.). Israel J. Plant Sci. 55: 137-145.

Tai T, Tanksley S. 1990. A rapid and inexpensive method for isolation of total DNA from dehydrated plant tissue. Plant Mol. Biol. Rep. 8: 297-303.

Talamè V, Bovina R, Sanguineti MC, Tuberosa R, Lundqvist U, Salvi S. 2008. TILLMore, a resource for the discovery of chemically induced mutants in barley. Plant Biotechnol. J. 6: 477-485.

Till BJ, Cooper J, Tai TH, Colowit P, Greene EA, Henikoff S, et al. 2007. Discovery of chemically induced mutations in rice by TILLING. BMC Plant Biol. 7: 19.

Tsai H, Howell T, Nitcher R, Missirian V, Watson B, Ngo KJ, et al. 2011. Discovery of rare mutations in populations: TILLING by sequencing. Plant Physiol. 156: 1257-1268.

Wei FJ, Droc G, Guiderdoni E, Hsing YI. 2013. International consortium of rice mutagenesis: Resources and beyond. Rice (N Y) 6: 39.

Wu JL, Wu C, Lei C, Baraoidan M, Bordeos A, Madamba $\mathrm{MR}$, et al. 2005. Chemical- and irradiation-induced mutants of indica rice IR64 for forward and reverse genetics. Plant Mol. Biol. 59: 85-97. 